\title{
Comportamento da pressão intra-ocular segundo os efeitos cardiorrespiratórios e hemodinâmicos induzidos pela anestesia com desflurano, em cães submetidos à hipovolemia experimental
}

\author{
Behavior of intraocularpressure according tocardiorespiratoryand hemodynamic \\ effects induced by desflurane in dogs subjected to experimental hypovolemia
}

Ivia Carmem Talieri ${ }^{1}$

Cristiane dos Santos Honsho ${ }^{2}$

Newton Nunes ${ }^{3}$

Almir Pereira de Souza ${ }^{4}$

Juan Carlos Duque ${ }^{5}$

\footnotetext{
${ }^{1}$ Médica Veterinária Doutoranda do Programa de Pósgraduação em Cirurgia Veterinária do Departamento de Clínica e Cirurgia Veterinária da Faculdade de Ciências Agrárias e Veterinárias - FCAV da Universidade Estadual Paulista (UNESP). Jaboticabal (SP).

${ }^{2}$ Médica Veterinária Doutoranda do Programa de Pósgraduação em Cirurgia Veterinária do Departamento de Clínica e Cirurgia Veterinária da FCAV da UNESP. Jaboticabal (SP)

Professor Assistente do Departamento de Clínica e Cirurgia Veterinária da FCAV da UNESP. Jaboticabal (SP)

${ }^{4}$ Médico Veterinário Doutorando do Programa de Pósgraduação em Cirurgia Veterinária do Departamento de Clínica e Cirurgia Veterinária da FCAV da UNESP. Jaboticabal (SP).

${ }_{5}^{5}$ Médico Veterinário Doutorando do Programa de Pósgraduação em Cirurgia Veterinária do Departamento de Clínica e Cirurgia Veterinária da FCAV da UNESP. Jaboticabal (SP).

Endereço para correspondência: Ivia Carmem Talieri Rua Men de Sá, 1899, casa 20 - Maringá (PR)

Cep 87005-010

E-mail: iviact@ig.com.br

Recebido para publicação em 18.03.2004

Versão revisada recebida em 05.04.2005

Aprovação em 30.05.2005

Fonte de auxílio à pesquisa: Fundação de Amparo à Pesquisa do Estado de São Paulo - FAPESP
}

\begin{tabular}{l} 
RESUMO \\
\hline Objetivo: Observar o comportamento da pressão intra-ocular, segundo os \\
efeitos cardiorrespiratórios e hemodinâmicos induzidos pela anestesia \\
geral com desflurano, em cães submetidos à hipovolemia experimental. \\
Métodos: Foram utilizados 18 cães, machos e fêmeas, com peso entre $10 \mathrm{e}$ \\
$15 \mathrm{~kg}$. A hipovolemia foi realizada retirando-se 40 ml de sangue/kg de peso. \\
A seguir, a anestesia foi induzida com desflurano através de máscara facial, \\
até que a intubação orotraqueal fosse permitida. A pressão intra-ocular foi \\
medida por tonometria de aplanação. Valores para frequiência cardíaca, \\
débito cardíaco, pressão arterial média, pressão venosa central e pressão \\
parcial de CO ao final da expiração e frequiência respiratória foram mensu- \\
rados. Os parâmetros da avaliação foram registrados após a instrumenta- \\
lização e antes de qualquer outro procedimento (T0), quinze minutos \\
depois da indução da hipovolemia experimental (T45) e após 30 minutos \\
da indução anestésica (T75). Resultados: A pressão intra-ocular apresen- \\
tou relação direta somente com a pressão parcial de CO no final da \\
expiração. Conclusões: Não foi possível estabelecer correlação entre \\
alterações da pressão arterial média e da pressão venosa central com a \\
pressão intra-ocular e houve relação direta entre os valores da pressão \\
intra-ocular e os de ETCO.
\end{tabular}

Descritores: Pressão intra-ocular/efeitos de drogas; Pressão intra-ocular/fisiologia; Anestesia/ veterinária; Olho/efeito de drogas; Humor aquoso/efeito de drogas; Anestésicos inalatórios; Hipovolemia/fisiopatologia; Hipovolemia/veterinária; Processos hemodinâmicos; Respiração/ efeito de drogas; Cães/cirurgia

\section{INTRODUÇÃO}

A pressão intra-ocular (Po) é a pressão exercida pelo conteúdo do olho contra a parede que o contém ${ }^{(1-2)}$, sendo determinada pela taxa de produção e drenagem de humor aquoso, pelo volume do vítreo, volume sangüíneo da coróide, rigidez da esclera, tensão do músculo orbicularis oculi e pela pressão externa $^{(1-6)}$. A compressão externa do globo, por meio da contração dos músculos extra-oculares, pode aumentar diretamente a Po ou pode ter efeitos indiretos, induzindo a alterações nos volumes dos componentes intra-oculares ${ }^{(1,4)}$.

A manutenção da Po abaixo dos limites normais é essencial para boa anestesia em cirurgias intra-oculares, visto que flutuações na Po podem ter efeitos desastrosos durante e após tais cirurgias ${ }^{(6-10)}$. 
522 Comportamento da pressão intra-ocular segundo os efeitos cardiorrespiratórios e hemodinâmicos induzidos pela anestesia com desflurano, em cães submetidos à hipovolemia experimental

Fármacos utilizados na anestesia podem afetar a Po agindo diretamente sobre o diencéfalo, facilitando ou inibindo a produção do humor aquoso, relaxando ou contraindo o músculo orbicularis e os músculos extra-oculares ou, ainda, indiretamente, por meio dos efeitos sobre os sistemas cardiovascular e respiratório ${ }^{(1,4,9)}$. Muitos autores enfatizam a importância dos anestésicos na cirurgia oftálmica e relatam a redução da Po com os agentes intravenosos e inalatórios ${ }^{(2,6)}$. Contudo, há relatos de que o desflurano e o sevoflurano não alteram a Po quando utilizados em cães normais, segundo estudo conduzido de acordo com a mesma metodologia utilizada na presente pesquisa $^{(11)}$.

A importância da pressão venosa central (PVC) sobre a Po é bem conhecida. Com uma elevação na PVC, a drenagem sangüínea do olho é inibida, resultando em aumento da Po. Pesquisadores observaram uma correlação significativa entre a PVC e a PIO em cães e gatos, respectivamente. Aumento ou diminuição da PVC provoca mudança paralela, de magnitude similar, na $\mathrm{Po}^{(1,12-13)}$.

Alterações na concentração de $\mathrm{CO}_{2}$ arterial provocam alterações semelhantes na Po e na PVC. A cessação de $\mathrm{CO}_{2}$ ou a hiperventilação induz à redução abrupta da Po e da PVC. Acredita-se que uma redução tão brusca na Po seja de natureza vascular, devido à alteração no volume sangüíneo, provavelmente relacionada à vasoconstrição dos vasos da corói$\mathrm{de}^{(1,14)}$. O mesmo estudo indica que o mecanismo de alteração na Po, induzido por $\mathrm{CO}_{2}$, seja predominantemente uma alteração na PVC que, via veia vórtex, altera o fluxo sangüíneo venoso coroidal e, portanto, a $\mathrm{Po}^{(14)}$.

O propósito deste estudo foi observar o comportamento da pressão intra-ocular, segundo os efeitos cardiorrespiratórios e hemodinâmicos induzidos pela anestesia geral com desflurano, em cães submetidos à hipovolemia experimental.

\section{MÉTODOS}

Os protocolos da pesquisa foram aprovados pela Comissão de Ética e Bem-Estar Animal da Faculdade de Ciências Agrárias e Veterinárias/UNESP - Campus de Jaboticabal (processo $n^{\circ}$ 003083). Outrossim, seguiram-se os cuidados bioéticos preconizados pela ARVO - Association for Research in Vision and Ophthalmology (National Institutes of Health $\mathrm{Pu}-$ blications No 85-23: Revised 1985) relativamente à utilização de animais em pesquisas oftálmicas.

Utilizaram-se 18 cães machos e fêmeas hígidos, sem raça definida, adultos e sadios, com peso corpóreo entre 10 e $15 \mathrm{~kg}$. Objetivando-se padronização e exclusão de variáveis indesejáveis, os animais foram examinados para avaliação das condições de higidez. Consideraram-se as semiotécnicas clínica, oftálmica e hematimétrica de rotina.

Na clínica, aferiram-se a temperatura retal, a coloração das mucosas aparentes, o turgor da pele (desidratação), o tempo de preenchimento capilar (TPC), a freqüência/ritmo cardíacos e a frequiência respiratória/sons pulmonares (auscultação), os linfonodos e o abdômen (palpação). Para a oftálmica, elencaram-se o teste da lágrima de Schirmer, a biomicroscopia em lâmpada de fenda, a tonometria de aplanação, a oftalmoscopia binocular indireta e a prova da fluoresceína. A avaliação hematimétrica constou de hemograma completo.

No dia anterior à experimentação, os cães foram anestesiados com propofol (Cristália, Produtos Químicos e Farmacêuticos Ltda., Itapira - SP, Brasil), na dose de $5 \mathrm{mg} / \mathrm{kg}$ por via intravenosa e, após tricotomia e anti-sepsia da face medial do membro pélvico, efetuou-se a abordagem cirúrgica para cateterização da artéria femoral. O cateter foi ocultado no espaço subcutâneo e a incisão fechada com fio de náilon em pontos simples interrompidos.

Decorridas 24 horas, os cães foram mantidos em decúbito lateral esquerdo para infiltração local com xilocaína (Xylestesin, Cristália, Produtos Químicos e Farmacêuticos Ltda., Itapira - SP, Brasil) na incisão. Após dez minutos, a sutura foi removida e o cateter intra-arterial foi exteriorizado e conectado ao transdutor do equipamento multiparamétrico (Dixtal 2010 LCD, Dixtal Biomédica, Manaus - AM, Brasil) para aferir a pressão arterial. Utilizando-se a veia femoral, introduziu-se cateter Swan-Ganz 5F (Swan Ganz cateter, Baxter, Sta Catarina - SP, Brasil) até a artéria pulmonar. O adequado posicionamento foi efetuado com o auxílio da leitura de onda de pulso observada no monitor multiparamétrico.

Em seguida, efetuou-se a remoção sangüínea ( $40 \mathrm{ml} / \mathrm{kg}$ ) pelo cateter colocado na artéria femoral, com velocidade não superior a $100 \mathrm{ml} / 5 \mathrm{~min}$. O sangue removido foi armazenado em bolsas para transfusão sangüínea e mantido em temperatura ambiente, para posterior re-infusão ao término do experimento.

Após 15 minutos da retirada do volume total de sangue (40 ml/kg), realizou-se a indução anestésica com desflurano (Suprane, Baxter Sta Catarina - SC, Brasil), administrado por máscara facial com fluxo diluente de oxigênio de $41 / \mathrm{min}$. Procedeu-se a intubação orotraqueal com sonda de Magil e a manutenção foi realizada com 1,5 CAM (aferida pela leitura no analisador de gases anestésicos (Ohmeda - Mod. 5250 RGM, Miami - Fl, USA) do anestésico diluído em 100\% de oxigênio, com fluxo de $30 \mathrm{ml} / \mathrm{kg}$, administrados em circuito anestésico tipo semifechado (Ohmeda - Mod. EXCEL 210 SE, Miami - Fl, USA), dotado de vaporizador específico para o agente anestésico empregado. Os animais foram mantidos sob respiração espontânea durante todo o procedimento.

A frequiência cardíaca (FC) e o ritmo cardíaco foram avaliados pelos traçados eletrocardiográficos, registrados por meio de eletrocardiografia computadorizada, em derivação II. A pressão arterial média (PAM) foi mensurada pela leitura da onda de pulso (obtida por meio do cateter na artéria femoral) no monitor do equipamento multiparamétrico.

O débito cardíaco (DC) foi aferido pela técnica de termodiluição descrita por NUNES em $2002^{(15)}$. A pressão venosa central (PVC) foi avaliada por meio do cateter de Swan-Ganz posicionado na veia cava, sendo a leitura aferida em monitor multiparamétrico digital. 
A frequiência respiratória (FR) e a pressão parcial de $\mathrm{CO}_{2}$, no final da expiração $\left(\mathrm{ETCO}_{2}\right)$ foram aferidas por meio de aparelho de ventilometria (Ventcare, Takaoka, São Paulo - SP, Brasil) e do analisador de gases, respectivamente.

As mensurações da pressão intra-ocular (Po), com tonômetro de aplanação (Tono pen XL, Mentor Medical Systems, Norwell - MA, EUA), foram realizadas em córneas dessensibilizadas com colírio de cloridrato de proximetacaína (Anestalcon, Alcon Laboratórios do Brasil, São Paulo - SP, Brasil). Os cães, mantidos em decúbito lateral esquerdo, eram posicionados em decúbito esternal, por ocasião das aferições das pressões intra-oculares, dos olhos direito e esquerdo.

Os parâmetros da avaliação foram registrados após a instrumentalização e antes de qualquer outro procedimento (T0), quinze minutos depois da indução da hipovolemia experimental (T45) e após 30 minutos da indução anestésica (T75), como ilustra a figura 1 .

As análises estatísticas foram realizadas segundo HOEL (1974), com o auxílio do programa InStat (Graphpad Instat:

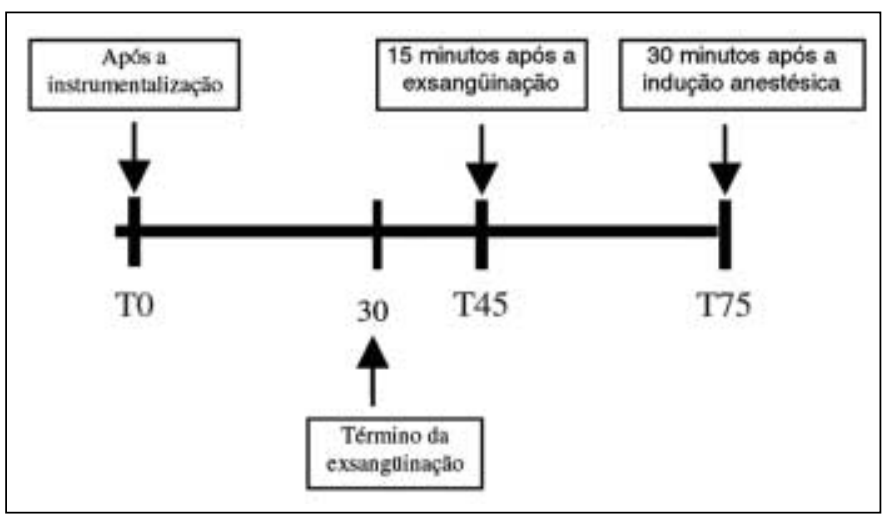

Figura 1 - Esquema do registro dos parâmetros de acordo com os momentos avaliados. TO $=$ início da hipovolemia; $30=30$ minutos, período da realização da hipovolemia; T45= 15 minutos decorridos da hipovolemia e início da anestesia com desflurano; T75= 30 minutos decorridos da anestesia com desflurano
GraphPad Software Oberlin, San Diego-CA, USA). Para detectar diferenças entre os momentos, dentro de cada grupo ao longo do tempo, foi utilizada a análise de variância de uma via para repetições múltiplas (ANOVA RM) seguida pelo teste de Tukey.

Os valores foram expressos em média \pm desvio padrão e considerados significativos quando $\mathrm{p}<0,05$.

\section{RESULTADOS}

Os valores encontrados para todos os parâmetros analisados em todos os momentos estão sumarizados na tabela 1 .

Com relação ao olho direito e esquerdo, não foram observadas alterações estatisticamente significativas da Po entre os momentos analisados: basal (T0), 15 minutos após hipovolemia (T45) e 30 minutos de anestesia por desflurano (T75). Confrontando-se os grupos entre si, também, não foram observadas diferenças nas pressões dos olhos direito e esquerdo (Figura 2A).

A respeito da $\mathrm{ETCO}_{2}$, observaram-se diferenças estatisticamente significativas no momento $\mathrm{T} 45$, comparativamente a T0 ( $\mathrm{p}<0,01)$ e no momento T75, comparativamente ao momento T45 (Figura 2B).

Comparando-se os valores da PAM entre os momentos, observaram-se diferenças significativas, com $\mathrm{p}<0,001$, para T45 e T75 comparativamente ao momento T0 e, $\mathrm{p}<0,01$ entre T45 e T75 (Figura 2C).

Quanto à PVC foram observadas diferenças estatisticamente significativas $(\mathrm{p}<0,01)$ nos momentos T45 e T75, comparativamente ao T0. Não foram observadas diferenças na comparação entre T45 e T75 (p>0,05) (Figura 2D).

Não houve diferença significativa na FC quando da comparação entre os momentos T0 e T45 e entre T45 e T75, observando-se porém, diferença significativa $(\mathrm{p}<0,05)$ entre T0 e T75 (Figura 2E).

Com relação ao DC foram observadas diferenças significativas $(\mathrm{p}<0,001)$ entre os momentos T45 e T75, comparativamente a T0 (Figura 2F).

\begin{tabular}{|c|c|c|c|}
\hline Parâmetro/Momento & TO & T45 & T75 \\
\hline PoD (mmHg) & $14,61 \pm 3,24$ & $14,06 \pm 3,89$ & $15,56 \pm 4,06$ \\
\hline PoE (mmHg) & $15,22 \pm 3,15$ & $13,61 \pm 4,82$ & $14,56 \pm 3,75$ \\
\hline $\mathrm{ETCO}_{2}(\mathrm{mmHg})$ & $29,39 \pm 6,62$ & $20,00 \pm 7,25^{a}$ & $35,06 \pm 11,51^{b}$ \\
\hline PAM $(\mathrm{mmHg})$ & $113,22 \pm 12,74$ & $62,61 \pm 17,86^{a}$ & $49,33 \pm 12,15^{\mathrm{ab}}$ \\
\hline PVC (mmHg) & $1,722 \pm 2,42$ & $-0,33 \pm 2,57^{a}$ & $-0,56 \pm 2,50^{a}$ \\
\hline FC (bpm) & $121,44 \pm 33,32$ & $135,11 \pm 40,69$ & $148,39 \pm 31,34^{a}$ \\
\hline $\mathrm{DC}(\mathrm{L} / \mathrm{min})$ & $2,59 \pm 0,79$ & $1,11 \pm 0,31^{a}$ & $1,10 \pm 0,35^{a}$ \\
\hline FR (ipm) & $23,94 \pm 11,57$ & $31,11 \pm 12,80$ & $19,56 \pm 8,70^{\mathrm{a}}$ \\
\hline \multicolumn{4}{|c|}{$\begin{array}{l}\mathrm{T} 0=\text { antes de qualquer procedimento; } \mathrm{T} 45=15 \text { minutos após a hipovolemia; } \mathrm{T} 75=30 \text { minutos após indução com desflurano; PoD= pressão intra-ocular do olho direito; } \\
\mathrm{PoE}=\text { pressão intra-ocular do olho esquerdo; } \mathrm{ETCO}_{2}=\text { pressão parcial de } \mathrm{CO}_{2} \text { no final da expiração; } \mathrm{PAM}=\text { pressão arterial média; } \mathrm{PVC}=\text { pressão venosa central; } \mathrm{FC}= \\
\text { freqüência cardíaca; } \mathrm{DC}=\text { débito cardíaco. Análises estatísticas - programa InStat (Graphpad Instat: GraphPad Software Oberlin, San Diego - CA, USA) e ANOVA } \\
\text { RM seguida pelo teste de Tukey. } \\
\text { aSignificativamente diferente de T0. 'Significativamente diferente de T45. }\end{array}$} \\
\hline
\end{tabular}


524 Comportamento da pressão intra-ocular segundo os efeitos cardiorrespiratórios e hemodinâmicos induzidos pela anestesia com desflurano, em cães submetidos à hipovolemia experimental

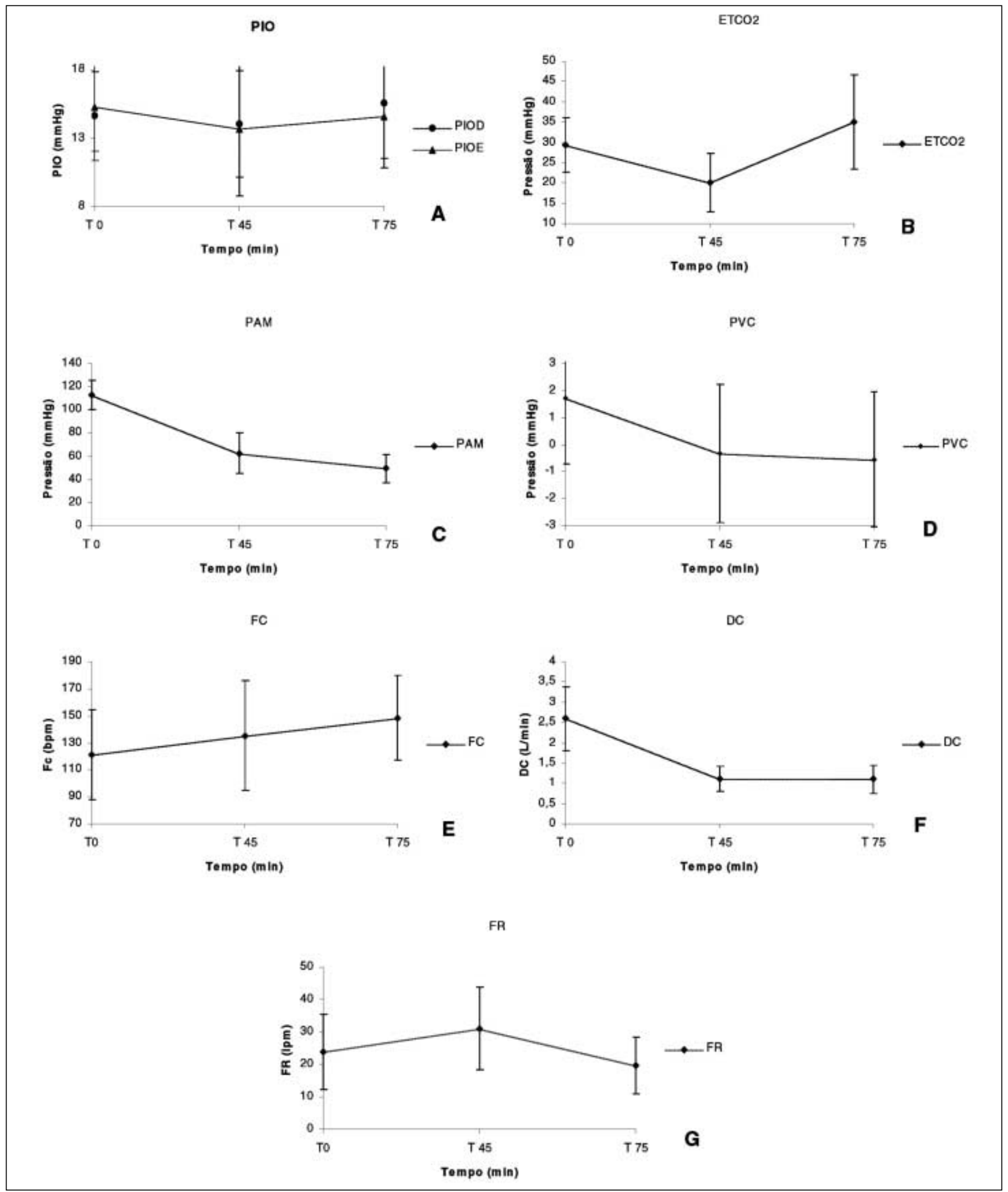

Figura 2 - Valores médios de A: pressão intra-ocular nos olhos direito (PIOD) e esquerdo (PIOE); B: pressão parcial de $\mathrm{CO}_{2}$ no final da expiração $\left(\mathrm{ETCO}_{2}\right)$; C: pressão arterial média (PAM); D: pressão venosa central (PVC); E: freqüência cardíaca (FC); F: débito cardíaco (DC) e G: freqüência respiratória (FR), em cães submetidos à hipovolemia experimental e anestesiados com desflurano

Arq Bras Oftalmol. 2005;68(4):521-6 
Na FR observou-se diferença estatisticamente significativa ( $<<0,01)$ no momento T75, comparativamente a T0 (Figura 2G).

\section{DISCUSSÃO}

Em estudo anterior, a hipovolemia em cães foi induzida com a retirada de $35,7 \pm 4,7 \mathrm{ml}$ de sangue $/ \mathrm{kg}$ de peso e os autores observaram, em adição às alterações imediatamente após hemorragia, aumento da FC após estabilização da PAM em $60 \mathrm{mmHg}^{(16)}$. Os efeitos cardiovasculares induzidos pela remoção desta quantidade de sangue foram similares àqueles já relatados na literatura ${ }^{(17)}$, após a remoção de $44 \mathrm{ml} / \mathrm{kg}$. Da mesma forma, retirando-se $40 \mathrm{ml}$ de sangue $/ \mathrm{kg}$ de peso foi possível observar neste estudo, o aumento da FC imediatamente após a hipovolemia.

Pôde-se observar ainda que a PAM sofreu acentuada queda, porém a Po diminuiu discretamente durante a hipovolemia. Dessa forma, não foi possível correlacionar a redução da PAM com os valores da Po. Os dados deste estudo corroboram com vários artigos na literatura que relatam a inexistência de relação entre a PAM e a Po. Por exemplo, Macri et al. (1961), observou em gatos, que a pressão nas artérias ciliares não estava associada com a pressão sangüínea sistêmica, e que havia dissociação entre as artérias ciliares e a artéria oftálmica, visto que a pressão da artéria oftálmica mantinha íntima relação com a pressão arterial sistêmica. ${ }^{(1,12-13,18)}$ Almeida et al. (2004), não observaram alterações significativas na PAM e na PVC, bem como na FC com a utilização de desflurano em cães normocapnéicos ${ }^{(11)}$. É interessante citar que o estudo de Almeida et al. (2004), foi realizado na mesma instituição (Faculdade de Ciências Agrárias e Veterinárias de Jaboticabal UNESP) que o presente trabalho, nas mesmas condições, no Laboratório de Anestesiologia.

Em humanos observou-se que a PAM elevada não alterava a Po, pois ocorria aumento na resistência vascular e a velocidade média de fluxo sangüíneo permanecia constante na artéria oftálmica e nas artérias ciliares ${ }^{(19)}$. Em suínos relata-se que a hipotensão arterial induzida por drogas não alterava a $\mathrm{Po}^{(20)}$. Alterações na pressão arterial sistêmica mostraram deprimir a formação do humor aquoso somente quando reduzida a níveis incompatíveis com a adequada perfusão tecidual ${ }^{(1,18)}$.

Por outro lado, a PAM relacionou-se indiretamente com a $\mathrm{ETCO}_{2}$, pois seu valor continuou em queda aos 30 minutos da indução com desflurano, enquanto o valor da $\mathrm{ETCO}_{2}$ aumentou. $\mathrm{O}$ valor crescente de $\mathrm{ETCO}_{2}$ promoveu uma tentativa compensatória de oxigenação sangüínea, por meio do aumento da FR.

Da mesma maneira, os valores de $\mathrm{ETCO}_{2}$ mantiveram correlação direta com os valores da Po. Outros autores já haviam relatado que o aumento da pressão arterial de $\mathrm{CO}_{2}\left(\mathrm{PaCO}_{2}\right)$

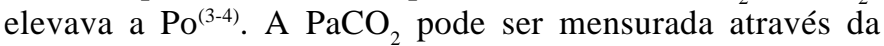
pressão alveolar de $\mathrm{CO}_{2}$ expirado $\left(\mathrm{ETCO}_{2}\right)^{(21)}$. As tensões de $\mathrm{CO}_{2}$ e $\mathrm{O}_{2}$, no sangue arterial, podem afetar a Po por meio de três mecanismos: a produção de humor aquoso, a vasodilatação intra ou extra-ocular e também o tônus dos músculos extra- oculares $^{(1-3,8,18)}$. O mesmo estudo evidenciou que a resistência vascular coroidal, em seu estudo, variou diretamente com a concentração de $\mathrm{CO}_{2}$ inalado ${ }^{(1)}$.

Alterações na $\mathrm{PaCO}_{2}$ são acompanhadas por alterações semelhantes na Po e na PVC, podendo-se concluir que o aumento da Po, após elevação da $\mathrm{ETCO}_{2}$, ocorre como resultado da vasodilatação coroidal ou elevação da PVC ou, provavelmente, pela combinação de ambas ${ }^{(1,2,14)}$.

Almeida et al. (2004), seguindo a metodologia utilizada nesta pesquisa, verificaram que a utilização de desflurano e sevoflurano, em cães normocapnéicos, mantém os valores de Po dentro da normalidade, tornando-os seguros para a utilização em cirurgias intra-oculares ${ }^{(11)}$.

Em 1961 foi sugerido que alterações na PVC resultavam em alterações correspondentes no diâmetro dos vasos sangüíneos intra-oculares, mais provavelmente nos vasos ciliares ${ }^{(13)}$. Afirmou-se também, que estas alterações no diâmetro dos vasos alteram a Po simplesmente por seus efeitos no volume intra-ocular. Contudo, neste estudo, os valores de PVC não mantiveram relação direta com o comportamento da Po.

A retirada de sangue da circulação resulta em aumento na utilização de oxigênio e diminuição da PAM, da PVC e do índice cardíaco $^{(16)}$. Estudos recentes dos efeitos da hemorragia aguda em coelhos e em cães não anestesiados demonstraram claramente uma resposta hemodinâmica bifásica, similar ao que ocorre em seres humanos. Durante a primeira fase, a pressão sangüínea é mantida por meio do aumento da resistência vascular periférica (RVP) e da frequiência cardíaca (FC), caracterizando um aumento da atividade simpática. Quando o volume sangüíneo é drasticamente reduzido, a PAM cai devido à diminuição da RVP, iniciando a segunda fase da resposta hemodinâmica, que é a inibição simpática, responsável pela hipotensão(22).

$\mathrm{Na}$ literatura pesquisada constataram que a PAM, a PVC, o débito cardíaco e o índice cardíaco diminuem com a indução da hipovolemia, ao contrário da FC e da RVP, que aumen$\operatorname{tam}^{(23)}$. Conforme as citações, com este estudo foi possível comprovar redução da PAM, da PVC, do débito cardíaco, da $\mathrm{ETCO}_{2}$ e da Po com a hipovolemia, bem como aumento compensatório da FR e da FC, antes da anestesia com desflurano.

Todavia, ainda não há registro do comportamento da Po durante a anestesia com desflurano em condições de hipovolemia e de sua correlação com os parâmetros cardiorrespiratórios e hemodinâmicos obtidos nesta situação. O presente estudo pode ser útil, como fonte de dados nunca descritos, para futuros trabalhos. Julgamos de importância, principalmente, o encontro da relação direta do comportamento da Po com o da $\mathrm{ETCO}_{2}$, mesmo na hipovolemia, e não com o da PVC, como descrito em vários trabalhos.

\section{CONCLUSÕES}

Permite-se concluir que:

Não foi possível estabelecer correlação entre alterações da PAM e da PVC com a Po;

Houve relação direta entre os valores da Po e os de $\mathrm{ETCO}_{2}$. 
526 Comportamento da pressão intra-ocular segundo os efeitos cardiorrespiratórios e hemodinâmicos induzidos pela anestesia com desflurano, em cães submetidos à hipovolemia experimental

\section{AGRADECIMENTOS}

Os autores agradecem à Fundação de Amparo à Pesquisa do Estado de São Paulo - FAPESP, pelo apoio financeiro na aquisição dos aparelhos: OHMEDA - Mod. 5250 RGM (Processo FAPESP 96/12830-0), OHMEDA - Mod. Excel 210 SE (Processo FAPESP 97/10668-4), TAKAOKA - Mod. Ventcare (Processo FAPESP 98/03153-0).

\section{ABSTRACT}

Purpose: To observe the behavior of intraocular pressure according to the cardiopulmonary and hemodynamic effects induced by desflurane in dogs subjected to experimental hypovolemia. Methods: Eighteen healthy male and female mongrel dogs, weighing between 10 and $15 \mathrm{~kg}$ were used. Hypovolemia was induced by withdrawal of $40 \mathrm{ml}$ blood/kg body weight. Then anesthesia was induced with desflurane by mask until tracheal intubation was permitted. Intraocular pressure was measured with applanation tonometry. Heart rate, cardiac output, mean arterial pressure, central venous pressure, endtidal concentration of $\mathrm{CO}_{2}$ and respiratory rate were recorded. Parameters were registered after animal instrumentation and before any procedure in the awake dogs (T0), fifteen minutes after experimental hemorrhage induction (T45), and after thirty minutes of desflurane anesthesia (T75). Results: Intraocular pressure presented direct correlation only with pressure and end-tidal concentration of $\mathrm{CO}_{2}$. Conclusions: It was not possible to establish a correlation between alterations of mean arterial pressure and central venous pressure and intraocular pressure and there was a direct relationship between values of intraocular pressure and values of exhaled $\mathrm{CO}_{2}$.

Keywords: Intraocular pressure/drug effects; Intraocular pressure/physiology; Anesthesia/veterinary; Eye/drug effects; Aqueous humor/drug effects; Anesthetics, inhalation; Hypovolemia/physiopathology; Hypovolemia/veterinary; Hemodynamic processes; Respiration/drug effects; Dogs/ surgery

\section{REFERÊNCIAS}

1. Murphy DF. Anesthesia and intraocular pressure. Anesth Analg. 1985;64(5): 520-30.

2. Cunnigham AJ, Barry P. Intraocular pressure - physiology and implications for anaesthetic management. Can Anaesth Soc J. 1986;33(2):195-208.

3. Duncalf D, Weitzner WS. Ventilation and hypercapnea on intraocular pressure during anesthesia. Anesth Analg. 1963;42(4):232-7.

4. Brunson DB. Anesthesia in ophthalmic surgery. Vet Clin North Am Small Anim Pract. 1980;10(2):481-95.

5. Artru AA. Rate of anterior chamber aqueous formation, trabecular outflow facility, and intraocular compliance during desflurane or halothane anesthesia in dogs. Anesth Analg 1995;81(3):585-90.

6. Sator S, Wildling E, Schabernig C, Akramian J, Zulus E, Winkler M Desflurane maintains intraocular pressure at an equivalent level to isoflurane and propofol during unstressed non-ophthalmic surgery. Br J Anaesth 1998 $80(2): 243-4$

7. Cameron AE. Intra-ocular pressure too low. An unusual anaesthetic hazard. Anaesthesia 1975;37(7):735-7.

8. Trim CM, Colbern GT, Martin CL. Effect of xylazine and ketamine on intraocular pressure in horses. Vet Rec, 1985;117(17):442-3.

9. Collins BK, Gross ME, Moore CP, Branson KR. Physiologic, pharmacologic, and practical considerations for anesthesia of domestic animals with eye disease. J Am Vet Med Assoc. 1995;207(2):220-30.

10. Nunes N, Laus JL. Técnicas anestésicas destinadas à cirurgia ocular no cão. Braz J Vet Res Anim Sci. 1995;32(3):177-80.

11. Almeida DE, Rezende ML, Nunes N, Laus JL. Evaluation of intraocular pressure in association with cardiovascular parameters in normocapnic dogs anesthetized with sevolfurane and desflurane. Vet Ophthalmol. 2004;7(4):265-9.

12. Macri FJ. Vascular pressure relationship and the intraocular pressure. Arch Ophthalmol. 1961;65(1):133-6.

13. Macri FJ. Interdependence of venous and eye pressure. Arch Ophthalmol 1961;65:442-9.

14. Hvidberg A, Kessing SV, Fernandes A. Effect of changes in $\mathrm{PCO}_{2}$ and body positions on intraocular pressure during general anesthesia. Acta Ophthalmol (Copenh). 1981;59(4):465-75.

15. Nunes N. Monitoração da anestesia. In: Fantoni DT, Cortopassi SR, editores. Anestesia em cães e gatos. São Paulo: Roca; 2002. p.64-81.

16. Ilkiw JE, Haskins SC, Patz JD. Cardiovascular and respiratory effects of thiopenta administration in hypovolemic dogs. Am J Vet Res. 1991;52(4): 576-80.

17. Kim SI, Desai JM, Shoemaker WC. Sequence of cardiorespiratory alterations after gradual prolonged hemorrhage in conscious dogs. Am J Physiol. 1969, 216(5):1044-50.

18. Gum GG, Gelatt KN, Ofri R. Physiology of the eye. In: Gelatt KN, editor. Veterinary ophthalmology. Philadelphia: Lippincott Williams e Wilkins; 1999. p.151-82.

19. Michelson G, Groh M, Gründler A. Regulation of ocular blood flow during increases of arterial blood pressure. Br J Ophthalmol. 1994;78(6):461-5.

20. Jantzen JP, Hennes HJ, Rochels R, Wallenfang T. Deliberate arterial hypotension does not reduce intraocular pressure in pigs. Anesthesiology. 1992;77(3): 536-40.

21. Reece WO. Respiração dos mamíferos. In: Swenson MJ, editor. Dukes fisiologia dos animais domésticos. Rio de Janeiro: Guanabara Koogan; 1984. p.199-222.

22. Schadt JC, Ludbrook J. Hemodynamic and neurohumoral responses to acute hypovolemia in conscious mammals. Am J Physiol. 1991;260(2 Pt 2):H305-18.

23. Errando CL, Valia JC, Sifre C, Moliner S, Gil F, Gimeno O, et al. [Cardiocirculatory effects of intravenous anesthetic induction in an experimental model of acute hypovolemia]. Rev Esp Anestesiol Reanim. 1998;45(8):333-9. Spanish.

\section{Nos artigios enviados para publicação, o nome dos autores e suas afiliações devem estar completos. Isso facilitará a indexação e os links com as bases de dados e o CV Lates.}

